\title{
Clinical Study \\ Pancreatic Resections in Renal Failure Patients: Is It Worth the Risk?
}

\author{
K. S. Norman, ${ }^{1}$ S. R. Domingo, ${ }^{1,2}$ and L. L. Wong ${ }^{1,3}$ \\ ${ }^{1}$ Department of Surgery, University of Hawaii John A. Burns School of Medicine, Honolulu, HI 96813, USA \\ ${ }^{2}$ Queens Medical Center, Honolulu, HI 96813, USA \\ ${ }^{3}$ University of Hawaii Cancer Center, Honolulu, HI 96813, USA \\ Correspondence should be addressed to L. L. Wong; hepatoma@aol.com
}

Received 18 September 2013; Revised 11 December 2013; Accepted 29 December 2013; Published 9 February 2014

Academic Editor: Christos G. Dervenis

Copyright (c) 2014 K. S. Norman et al. This is an open access article distributed under the Creative Commons Attribution License, which permits unrestricted use, distribution, and reproduction in any medium, provided the original work is properly cited.

Background. Chronic kidney disease affects 20 million US patients, with nearly 600,000 on dialysis. Long-term survival is limited and the risk of complex pancreatic surgery in this group is questionable. Previous studies are limited to case reports and small case series and a large database may help determine the true risk of pancreatic surgery in this population. Methods. The American College of Surgeons National Surgical Quality Improvement Program database was queried (2005-2011) for patients who underwent pancreatic resection. Renal failure was defined as the clinical condition associated with rapid, steadily increasing azotemia (rise in BUN) and increasing creatinine above $3 \mathrm{mg} / \mathrm{dL}$. Operative trends and short-term outcomes were reviewed for those with and without renal failure (RF). Results. In 18,533 patients, 28 had RF. There was no difference in wound infections, neurologic or cardiovascular complications. Compared to non-RF patients, those with RF had more unplanned intubation (OR 4.89, 95\% CI 1.85-12.89), bleeding requiring transfusion (OR 3.12, 95\% CI 1.37-14.21), septic shock (OR 8.86, 95\% CI 3.75-20.91), higher 30 -day mortality $(21.4 \%$ versus $2.3 \%, P<0.001)$ and longer hospital stay (23 versus 12 days, $P<0.001)$. Conclusions. RF patients have much higher morbidity and mortality after pancreatic resections and surgeons should consider this before proceeding.

\section{Introduction}

Although the incidence of end stage renal disease (ESRD) has remained relatively stable in the United States, the prevalence has increased, such that 593,000 people are currently living on hemodialysis, on peritoneal dialysis, or with a functioning kidney transplant. Improvements in renal-replacement therapy, overall treatment, and access to care have increased patient survival in ESRD. In the prevalent population, mortality rates have declined by nearly 25 percent over the last two decades. Patients are living longer on renal replacement therapy and the population currently on dialysis is increasing in age. The overall incidence of dialysis patients is 340 per million persons but since 2000 , the adjusted incidence rate of ESRD has increased by $12.2 \%$ for those patients 75 years or older (1773 per million persons) $[1,2]$.
Because of the rising number of aging dialysis patients, there will likely be an increased need for use of hospital resources and cancer care in this population. Multiple studies have consistently showed increased complications and mortality in renal patients undergoing general, vascular, and cardiac surgical procedures [3-8]. Patient selection and vigilant management are recommended in this population.

The question of whether patients with renal failure should undergo major cancer surgery has not been specifically addressed in the medical literature. The complexity of the surgery and overall prognosis of the type of cancer involved will need to be considered in choosing options for these patients. Few studies address outcomes of pancreatic surgery in patients with renal dysfunction and all were single center studies consisting of small series of patients [9-11]. The purpose of this study is to utilize a large, nationwide database 
TABLE 1: Patient characteristics.

\begin{tabular}{|c|c|c|c|c|}
\hline & Total $(n=18533)$ & Group RF $(n=28)$ & Group non-RF $(n=18505)$ & $P$ value \\
\hline Mean age (SD) & $61.8(13.9)$ & $67.9(13.5)$ & $61.8(13.9)$ & $P=0.02$ \\
\hline Age 65 or older (\%) & $8659(46.7 \%)$ & $16(57.1 \%)$ & $8627(46.6 \%)$ & NS $(P=0.34)$ \\
\hline Males (\%) & $8922(48.1 \%)$ & $17(60.7 \%)$ & $8905(48.1 \%)$ & NS $(P=0.41)$ \\
\hline Diabetes & $4197(22.6 \%)$ & $11(39.3 \%)$ & $4186(22.6 \%)$ & $P=0.040$ \\
\hline History of smoking & $4065(21.9 \%)$ & $6(21.4 \%)$ & $4059(21.9 \%)$ & NS $(P=0.95)$ \\
\hline History of alcohol use & $478(2.6 \%)$ & 0 & $478(2.6 \%)$ & NS $(P=0.45)$ \\
\hline History of COPD & $815(4.4 \%)$ & $3(10.7 \%)$ & $812(4.4 \%)$ & NS $(P=0.12)$ \\
\hline Myocardial infarction within 6 mo. & $56(0.3 \%)$ & $3(10.7 \%)$ & $53(0.3 \%)$ & $P<0.0001$ \\
\hline Hypertension requiring medications & $9620(51.9 \%)$ & $24(85.7 \%)$ & $9596(51.9 \%)$ & $P<0.0001$ \\
\hline Congestive heart failure & $51(0.3 \%)$ & 0 & $51(0.3 \%)$ & NS $(P=0.86)$ \\
\hline Transient ischemic attack & $338(1 / 8 \%)$ & $1(3.6 \%)$ & $337(1.8 \%)$ & NS $(P=0.55)$ \\
\hline Cerebrovascular disease & $255(1.4 \%)$ & $1(3.6 \%)$ & $254(1.4 \%)$ & NS $(P=0.43)$ \\
\hline Currently on steroids & $383(2.1 \%)$ & $3(10.7 \%)$ & $380(2.1 \%)$ & $P=0.02$ \\
\hline Bleeding disorder & $507(2.7 \%)$ & $6(21.4 \%)$ & $501(2 / 7 \%)$ & $P<0.0001$ \\
\hline Disseminated cancer & $617(3.3 \%)$ & $6(21.4 \%)$ & $611(3.3 \%)$ & $P<0.0001$ \\
\hline Prior radiation therapy & $376(2.0 \%)$ & 0 & $376(2.0 \%)$ & NS $(P=0.48)$ \\
\hline Prior chemotherapy & $350(1.9 \%)$ & $2(7.1 \%)$ & $348(1.9 \%)$ & NS $(P=0.09)$ \\
\hline Transfusion before surgery & $149(0.8 \%)$ & $3(10.7 \%)$ & $146(0.8 \%)$ & $P=0.001$ \\
\hline
\end{tabular}

to determine outcomes of pancreatic resection in patients with renal failure to assist surgeons and oncologists in selecting the optimal patients for these procedures.

\section{Materials and Methods}

Data for this study was obtained from the American College of Surgeons National Surgical Quality Improvement Program (ACS NSQIP). ACS NSQIP is a prospective, multiinstitutional, and clinical registry created by the Veterans Health Administration in 1994 for quality improvement purposes. Over 130 preoperative variables through 30 -day postoperative variables are collected on randomly assigned patients, including patient demographics, surgical profile, preoperative risk assessment, laboratory values, operative information, and 30-day morbidity and mortality rates. A highly trained Surgical Clinical Reviewer (SCR) collects the data. All reviewers receive extensive initial training prior to starting data collection and ongoing training via continuing education. ACS NSQIP monitors accrual rates and data sampling methodologies and conducts audits on a random basis, ensuring highly reliable data [12].

ACS NSQIP participant files for the years 2005-2011 were reviewed and Current Procedure Terminology (CPT) codes were used to identify all patients who underwent pancreatic procedures (48100-48999). We excluded pancreatic biopsy and pancreatic debridement as these were presumably done for unresectable pancreatic malignancies or necrotizing pancreatitis. From the remaining pancreatic cases, two groups were created: patients who had pancreatic resection with renal failure (group RF) and those who underwent pancreatic resection with normal renal function (group non-RF).

Renal failure was defined as an increased creatinine above $3 \mathrm{mg} / \mathrm{dL}$ on laboratory studies within 24 hours prior to surgery and patients on dialysis. Dialysis dependent patients were those with acute or chronic renal failure requiring peritoneal dialysis, hemodialysis, hemofiltration, hemodiafiltration, or ultrafiltration within two weeks prior to surgery.

Patient demographics included sex, age, smoking, and alcohol use. The comorbidities considered were diabetes, chronic obstructive pulmonary disease (COPD), congestive heart failure $(\mathrm{CHF})$, hypertension requiring medications, disseminated cancer, and transfusions within 3 days prior to surgery. Postoperative complications of interest were superficial surgical site infection, deep incisional surgical site infection, organ space surgical site infection, wound disruption, pneumonia, urinary tract infections, unplanned intubation, pulmonary embolism, deep vein thrombosis, cardiac arrest requiring cardiopulmonary resuscitation, myocardial infarction, intraoperative or postoperative transfusions, sepsis, and septic shock.

Finally other outcome measures reviewed included operative time, return to the operating room, hospital length of stay, 30-day mortality, and time from operation to death in those patients who expired.

2.1. Statistical Analysis. RF versus non-RF groups were compared for baseline characteristics. Numerical values were compared using Student $t$-test. Categorical and dichotomous variables were compared using chi-square testing. Associated risks were expressed as odds ratios (OR) with a 95\% confidence interval (CI). All reported $P$ values are two-tailed, and for all tests, $P<0.05$ was considered statistically significant.

With nominal regression, we used preoperative variables of sex, age > 65 years, history of diabetes, smoking, congestive heart failure, use of steroids, albumin $<3.0 \mathrm{gm} / \mathrm{dL}$, bilirubin > $2.0 \mathrm{mg} / \mathrm{dL}$, AST > $90 \mathrm{U} / \mathrm{L}$, hematocrit < 30\%, platelet count $<200 \times 10^{3} / \mathrm{cc}$, and prothrombin time $>14$ 
TABle 2: Preoperative laboratory studies. All values with standard deviation.

\begin{tabular}{lccc}
\hline & Group RF $(n=28)$ & Group non-RF $(n=18505)$ & $P$ value \\
\hline Sodium $(\mathrm{mmol} / \mathrm{L})$ & $138.1(3.8)$ & $138.9(3.2)$ & NS $(P=0.187)$ \\
BUN $(\mathrm{mg} / \mathrm{dL})$ & $45.2(22.0)$ & $14.8(7.3)$ & $P<0.0001$ \\
Creatinine $(\mathrm{mg} / \mathrm{dL})$ & $4.0(2.03)$ & $0.94(0.5)$ & $P<0.0001$ \\
Albumin $(\mathrm{gm} / \mathrm{dL})$ & $3.0(0.9)$ & $3.8(0.7)$ & $P<0.0001$ \\
Total bilirubin $(\mathrm{mg} / \mathrm{dL})$ & $2.0(3.19)$ & $1.57(2.51)$ & NS $(P=0.42)$ \\
Alanine aminotransferase (U/L) & $92.4(145.0)$ & $49.7(67.7)$ & $P=0.002$ \\
Alkaline phosphatase $(\mathrm{U} / \mathrm{L})$ & $234.3(266.3)$ & $162.4(161.0)$ & $P=0.026$ \\
WBC $(\times 108 / \mathrm{L})$ & $10.3(7.23)$ & $7.4(2.9)$ & $P<0.0001$ \\
Hematocrit $(\%)$ & $30.8(5.6)$ & $38.0(5.1)$ & $P<0.0001$ \\
Platelet count $\left(\times 10^{3} / \mathrm{mL}\right)$ & $265.4(97.0)$ & $P=0.012$ \\
Partial thromboplastin time (seconds) & $218.5(107.9)$ & $29.6(5.8)$ & $P=0.004$ \\
Prothrombin time $($ seconds) & $33.2(9.6)$ & $12.6(2.4)$ & $P<0.0001$ \\
\hline
\end{tabular}

TABle 3: Postoperative complications.

\begin{tabular}{|c|c|c|c|c|}
\hline & Total $(n=18533)$ & Group RF $(n=28)$ & Group non-RF $(n=18505)$ & $P$ value \\
\hline Superficial/skin infection & $1524(8.2 \%)$ & $1(3.6 \%)$ & $1523(8.2 \%)$ & NS $(P=0.37)$ \\
\hline Deep surgical site infection & $361(1.9 \%)$ & $1(3.6 \%)$ & $360(1.0 \%)$ & NS $(P=0.53)$ \\
\hline Intra-abdominal infection & $1887(10.2 \%)$ & $2(7.1 \%)$ & $1885(10.2 \%)$ & $\mathrm{NS}(P=0.60)$ \\
\hline Wound dehiscence & $271(1.5 \%)$ & 0 & $271(1.5 \%)$ & $\mathrm{NS}(P=0.52)$ \\
\hline Postoperative pneumonia & $888(4.8 \%)$ & $1(3.6 \%)$ & $887(4.8 \%)$ & $\mathrm{NS}(P=0.76)$ \\
\hline Need for reintubation & $793(4.3 \%)$ & $5(17.9 \%)$ & $788(4.3 \%)$ & $P<0.0001$ \\
\hline Pulmonary embolism & $210(1 / 1 \%)$ & 0 & $210(1.1 \%)$ & $\mathrm{NS}(P=0.57)$ \\
\hline Urinary tract infection & $934(5.0 \%)$ & $1(3.6 \%)$ & $933(5.0 \%)$ & $\mathrm{NS}(P=0.72)$ \\
\hline Myocardial infarction & $115(0.6 \%)$ & 0 & $115(0.6 \%)$ & $\mathrm{NS}(P=0.68)$ \\
\hline Cardiac arrest & $204(1.1 \%)$ & $3(10.7 \%)$ & $201(1.1 \%)$ & $P<0.0001$ \\
\hline Cerebrovascular accident & $50(0.3 \%)$ & 0 & $50(0.3 \%)$ & NS $(P=0.78)$ \\
\hline Deep venous thrombosis & $416(2.2 \%)$ & $2(7.1 \%)$ & $414(2.2 \%)$ & $\mathrm{NS}(P=0.08)$ \\
\hline Bleeding requiring transfusion & $2108(11.4 \%)$ & $8(28.6 \%)$ & $2100(11.3 \%)$ & $P=0.004$ \\
\hline Sepsis & $1854(10.0 \%)$ & $1(3.6 \%)$ & $1853(10.0 \%)$ & $\mathrm{NS}(P=0.26)$ \\
\hline Septic shock & $678(3.7 \%)$ & $7(25.0 \%)$ & $671(3.7 \%)$ & $P<0.0001$ \\
\hline Return to OR & $1141(6.2 \%)$ & $4(14.3 \%)$ & $1137(6.2 \%)$ & $\mathrm{NS}(P=0.08)$ \\
\hline
\end{tabular}

seconds and the presence of renal failure to determine if any of these factors were associated with 30-day mortality.

\section{Results}

During the 7-year period, 2005-2011, there were 18,533 patients who underwent pancreatic resection. Males to females were 8922 to 9585 and mean age was 61.8 years. Of this entire cohort, 28 patients were identified as having renal failure. Specific procedures included partial removal of pancreas (13), pancreatectomy (6), pancreatectomy with pancreatojejunotomy (3), distal pancreatectomy (3), and proximal pancreatectomy (3).

Patient demographics and comorbidities are as listed in Table 1. RF patients were older and were more likely to have diabetes, myocardial infarction within the previous 6 months, hypertension requiring medications, use of steroids, bleeding disorder, disseminated cancer, and a blood transfusion prior to surgery.

Preoperative laboratory studies are as shown in Table 2. RF patients had significantly worse initial bleeding parameters with higher protime and partial thromboplastin time, as well as a lower platelet count. Initial WBC and hematocrit were also lower in RF patients. RF patients had similar bilirubin but higher AST, alkaline phosphatase, and lower albumin prior to pancreas surgery.

Postoperative complications and outcome are detailed in Tables 3 and 4. Most notably, RF patients were more likely to need reintubation, have a cardiac arrest, and have bleeding that required blood transfusion. Although RF patients were not any more likely to have surgical site infections, wound infections, or sepsis, they were more likely to have septic shock. RF patients were more likely to require a return to the operating room. Hospital length of stay and 30-day mortality were significantly higher in RF patients. Of all patients who 
TABLE 4: Outcome measures.

\begin{tabular}{lccc}
\hline & Group RF $(n=28)$ & Group non-RF $(n=18505)$ & $P$ value \\
\hline Operative time in minutes (SD) & $316(139.7)$ & $324(142.7)$ & NS $(P=0.913)$ \\
Hospital length of stay in days (SD) & $23(25.7)$ & $12.0(11.0)$ & $P<0.001$ \\
Days from operation to death (SD) & $7.83(7.36)$ & $13.3(8.54)$ & NS $(P=0.36)$ \\
30-day mortality & $6 / 28(21.4 \%)$ & $425 / 18505(2.3 \%)$ & $P<0.001$ \\
\hline
\end{tabular}

TABle 5: Predictors of 30-day mortality.

\begin{tabular}{lcc}
\hline & Odds ratio (95\% CI) & $P$ value \\
\hline Renal failure & $6.13(2.32-16.18)$ & $P<0.001$ \\
History of congestive heart & $4.72(2.13-10.50)$ & $P<0.001$ \\
failure & $2.44(1.98-3.01)$ & $P<0.001$ \\
Age 65 or higher & $1.94(1.47-2.56)$ & $P<0.001$ \\
Albumin $<3.0$ gm/dL & $1.93(1.18-3.16)$ & $P=0.009$ \\
History of steroid use & $1.69(1.24-2.28)$ & $P=0.001$ \\
Hematocrit $<30 \%$ & $1.59(1.25-2.02)$ & $P<0.001$ \\
Protime $>14$ seconds & $1.33(1.08-1.64)$ & $P=0.008$ \\
Diabetes & $1.02(0.79-1.32)$ & $\mathrm{NS}(P=0.87)$ \\
Bilirubin $>2.0 \mathrm{mmol} / \mathrm{L}$ & &
\end{tabular}

died in this study, those with RF expired in a mean of 7.8 days compared to 13.3 days in non-RF patients.

Using nominal regression, the factors predictive of 30 day mortality included age 65 or higher, presence of diabetes, history of CHF, steroid use, albumin $<3.0 \mathrm{gm} / \mathrm{dL}$, bilirubin $>$ $2.0 \mathrm{mg} / \mathrm{L}, \mathrm{Hct}<30 \%$, protime $>14$ seconds, and renal failure (see Table 5). The presence of RF had the highest odds ratio at 6.13 .

\section{Discussion}

Advances in renal replacement therapy and better management of diabetes and cardiovascular problems have allowed patients with RF to live longer with their chronic illnesses, but they now may be living long enough to develop neoplasms that require surgical intervention. While previous large studies have addressed surgical procedures in dialysis patients, less is known about how these RF patients fare in pancreatic resections.

Previous studies have demonstrated increased surgical morbidity and mortality in patients with renal failure who are dialysis dependent. In the largest study, Gajdos et al. used NSQIP to investigate the effect of long-term dialysis on general surgical procedures. The 1506 dialysis patients had $12.7 \%$ mortality (30-day) compared to $1.5 \%$ in the 164,094 patients with normal renal function. Dialysis patients also had a higher pulmonary complications, reoperations, cardiovascular complications, surgical site infections, and hospital length of stay. While this study had a large number of dialysis patients, it included a variety of general surgical procedures with varying levels of complexity and is done for both benign and malignant reasons [7].
Pancreatic surgeries are among the most complex of the abdominal procedures. Many factors contribute to this risk but the effect of renal dysfunction has been variably reported. Age has been the most commonly cited risk factor for outcome in pancreatic surgery [13-16]. Diabetes, the reason for pancreatic resection (benign versus malignant), hospital volume, preoperative biliary drainage, and resection of other organs have all been mentioned as contributing to prognosis $[9,11,17-19]$. Renal failure and dysfunction have been shown to contribute to poor outcome in pancreatic head resections in 3 single center studies, but these studies had 300 cases or fewer in each series [9-11].

Two studies attempted to combine multiple risk factors in developing tools to assess patients' risk for pancreatic surgery. Are et al. using the Nationwide Inpatient Sample (NIS) database developed a nomogram to predict outcome for patients undergoing pancreatic resection for malignancy. Renal failure was the factor with the highest number points assigned in the nomogram [20]. On the other hand, Parikh et al. using NSQIP developed a risk calculator to predict outcome after pancreatic resection. Renal failure was not mentioned as a risk factor, but American Society of Anesthesiologists (ASA) classification, functional health status, sepsis, surgical extent, age, dyspnea, body mass index, coronary heart disease, gender, and bleeding disorder were the most important [21].

Two additional studies reviewed outcomes after pancreatic surgery utilizing the NIS and discussed the effect of renal failure. McPhee analyzed in-hospital mortality in patients who underwent pancreatectomy for neoplasm between 1998 and 2003. Of 39,463 patients, 395 (1\%) had renal failure and mortality rate of RF patients was $36.1 \%$ compared to the overall mortality rate of $5.9 \%$ [22]. Teh et al. evaluated the NIS database for major pancreatic resections done for benign and malignant disease between the years of 1988 and 2003. Among the cohort of 103,222 patients, $1 \%$ had renal insufficiency and this patient subset had increased in-hospital mortality (OR 6.3), perioperative complications (OR 2.3), and increased mortality following a major complication (OR 3.5) [23]. While these studies are helpful in stratifying renal failure as a risk, the clinical variables and specific outcomes in these RF patients are not described in detail.

Our study clearly demonstrates that renal failure increased morbidity and mortality for patients undergoing pancreatic resection. Although only a cohort of 28 patients, $21 \%$ of these patients died in a mean of 7.8 days after surgery. Renal failure increased the 30-day mortality by 6 -fold. Patients with renal failure also had a significantly higher rate of postoperative complications with an increased risk 
of unplanned reintubation, cardiac arrest, septic shock, and need for transfusion. Those patients who did survive remained hospitalized for almost twice as long as patients without RF.

This study did have limitations, as it utilized an administrative database and can only be as precise as the trained staff who enter the data. Although the number of pancreatic resections was large, the number of cases with RF was relative small and the data may have diminished accuracy especially in subcategories and with some of the laboratory values. In addition, NSQIP is able to define a preoperative creatinine but cannot tell us if creatinine is due to a chronic kidney disease or if this is an acute or relatively recent renal dysfunction. One might assume that a patient with acute progressive renal failure would not be subjected to a major pancreatic resection, but unfortunately this study cannot provide us with the exact information. The grouping of the procedures by NSQIP based on procedural codes was quite nonspecific and there was likely variability in coding by the staff. It is likely that "partial removal of pancreas" may have included both distal pancreatic resections and pancreaticoduodenectomies with varying extents of each procedure. A major limitation of the study is that NSQIP database did not report the exact pathology of the resected specimen or the details of the operative procedure. It is unclear if some of these cases represented benign or premalignant lesions such as cystic tumors or neuroendocrine tumors which may have significantly less risk and better short, and long-term outcome. Finally, although NSQIP can identify general complications, it is not a database that can delineate procedure-specific complications so important complications such as pancreatic leaks and fistulas could not be assessed.

\section{Conclusions}

In conclusion, this study establishes a significant negative effect of renal failure on outcome in pancreatic resections. It will be difficult to acquire larger cohorts of patients with this particular problem and NSQIP is really not able to assess risk based on specific diagnoses requiring pancreatic resection. Each RF patient should be assessed for overall risk and especially in the context of expected long-term survival based on the specific pancreatic diagnosis. Those patients who opt for pancreatic resection should be optimized in terms of bleeding parameters and should be monitored carefully for respiratory, bleeding, and septic complications. Strong consideration should be made for nonoperative therapies or no treatment in those patients who have prohibitive risk or limited long-term survival.

\section{Disclosures}

Dr. L. L. Wong is supported by Bayer Healthcare (Speakers Bureau). Drs. S. R. Domingo and K. S. Norman have no financial disclosures. This paper was not presented at any meeting or previous communication.

\section{Conflict of Interests}

The authors declare that there is no conflict of interests regarding the publication of this paper.

\section{Acknowledgment}

The authors wish to thank Wanda Muranaka and Dr. Whitney Limm for assistance with this project.

\section{References}

[1] United States Renal Data System, USRDS 2012 Annual Data Report: Atlas of Chronic Kidney Disease and End-Stage Renal Disease in the United States, National Institutes of Health, National Institute of Diabetes and Digestive and Kidney Diseases, Bethesda, Md, USA, 2012.

[2] Kidney Diseases Statistics for the United States, NIH Publication No. 12-3895, National Kidney and Urologic Diseases Information Clearinghouse, Bethesda, Md, USA, 2012.

[3] C. Gajdos, M. T. Hawn, D. Kile et al., "The risk of major elective vascular surgical procedures in patients with end-stage renal disease," Annals of Surgery, vol. 257, no. 4, pp. 766-773, 2013.

[4] L. Labrousse, C. de Vincentiis, F. Madonna, C. Deville, X. Roques, and E. Baudet, "Early and long term results of coronary artery bypass grafts in patients with dialysis dependant renal failure," European Journal of Cardio-Thoracic Surgery, vol. 15, no. 5, pp. 691-696, 1999.

[5] J. F. M. Bechtel, C. Detter, T. Fischlein et al., "Cardiac surgery in patients on dialysis: decreased 30-day mortality, unchanged overall survival," Annals of Thoracic Surgery, vol. 85, no. 1, pp. 147-153, 2008.

[6] S. Drolet, A. R. MacLean, R. P. Myers, A. A. M. Shaheen, E. Dixon, and W. D. Buie, "Morbidity and mortality following colorectal surgery in patients with end-stage renal failure: a population-based study," Diseases of the Colon and Rectum, vol. 53, no. 11, pp. 1508-1516, 2010.

[7] C. Gajdos, M. T. Hawn, D. Kile, T. N. Robinson, and W. G. Henderson, "Risk of major nonemergent inpatient general surgical procedures in patients on long-term dialysis," Archives of Surgery, vol. 15, pp. 1-7, 2012.

[8] C. R. Schneider, W. Cobb, S. Patel, D. Cull, C. Anna, and R. Roettger, "Elective surgery in patients with end stage renal disease: what's the risk?" The American Surgeon, vol. 75, no. 9, pp. 790-793, 2009.

[9] U. Adam, F. Makowiec, H. Riediger, W. D. Schareck, S. Benz, and U. T. Hopt, "Risk factors for complications after pancreatic head resection," The American Journal of Surgery, vol. 187, no. 2, pp. 201-208, 2004.

[10] J. P. Lerut, P. R. Gianello, J. B. Otte, and P. J. Kestens, "Pancreaticoduodenal resection. Surgical experience and evaluation of risk factors in 103 patients," Annals of Surgery, vol. 199, no. 4, pp. 432-437, 1984.

[11] D. J. Gouma, R. C. I. van Geenen, T. M. van Gulik et al., "Rates of complications and death after pancreaticoduodenectomy: risk factors and the impact of hospital volume," Annals of Surgery, vol. 232, no. 6, pp. 786-795, 2000.

[12] ACS NSQIP, Data Collection, Analysis and Reporting, ACS NSQIP, Chicago, Ill, USA, http://site.acsnsqip.org.

[13] T. S. Riall, "What is the effect of age on pancreatic resection?" Advances in Surgery, vol. 43, no. 1, pp. 233-249, 2009. 
[14] M. P. Spencer, M. G. Sarr, and D. M. Nagorney, "Radical pancreatectomy for pancreatic cancer in the elderly. Is it safe and justified," Annals of Surgery, vol. 212, no. 2, pp. 140-143, 1990.

[15] M. Oliverius, Z. Kala, M. Varga, R. Gürlich, V. Lanska, and H. Kubesova, "Radical surgery for pancreatic malignancy in the elderly," Pancreatology, vol. 10, no. 4, pp. 499-502, 2010.

[16] T. S. Riall, D. M. Reddy, W. H. Nealon, and J. S. Goodwin, “The effect of age on short-term outcomes after pancreatic resection: a population-based study," Annals of Surgery, vol. 248, no. 3, pp. 459-467, 2008.

[17] R. M. Cannon, R. LeGrand, R. B. Chagpar et al., "Multi-institutional analysis of pancreatic adenocarcinoma demonstrating the effect of diabetes status on survival after resection," $H P B$, vol. 14, no. 4, pp. 228-235, 2012.

[18] F. Maire, P. Hammel, B. Terris et al., "Prognosis of malignant intraductal papillary mucinous tumours of the pancreas after surgical resection. Comparison with pancreatic ductal adenocarcinoma," Gut, vol. 51, no. 5, pp. 717-722, 2002.

[19] F. P. Herter, A. M. Cooperman, T. N. Ahlborn, and C. Antinori, "Surgical experience with pancreatic and periampullary cancer," Annals of Surgery, vol. 195, no. 3, pp. 274-281, 1982.

[20] C. Are, C. Afuh, L. Ravipati, A. Sasson, F. Ullrich, and L. Smith, "Preoperative nomogram to predict risk of perioperative mortality following pancreatic resections for malignancy," Journal of Gastrointestinal Surgery, vol. 13, no. 12, pp. 2152-2162, 2009.

[21] P. Parikh, M. Shiloach, M. E. Cohen et al., "Pancreatectomy risk calculator: an ACS-NSQIP resource," $H P B$, vol. 12, no. 7, pp. 488-497, 2010.

[22] J. T. McPhee, J. S. Hill, G. F. Whalen et al., "Perioperative mortality for pancreatectomy: a national perspective," Annals of Surgery, vol. 246, no. 2, pp. 246-253, 2007.

[23] S. H. Teh, B. S. Diggs, C. W. Deveney, and B. C. Sheppard, "Patient and hospital characteristics on the variance of perioperative outcomes for pancreatic resection in the United States: a plea for outcome-based and not volume-based referral guidelines," Archives of Surgery, vol. 144, no. 8, pp. 713-721, 2009. 


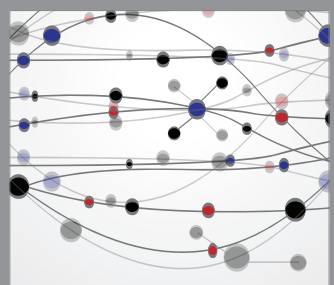

The Scientific World Journal
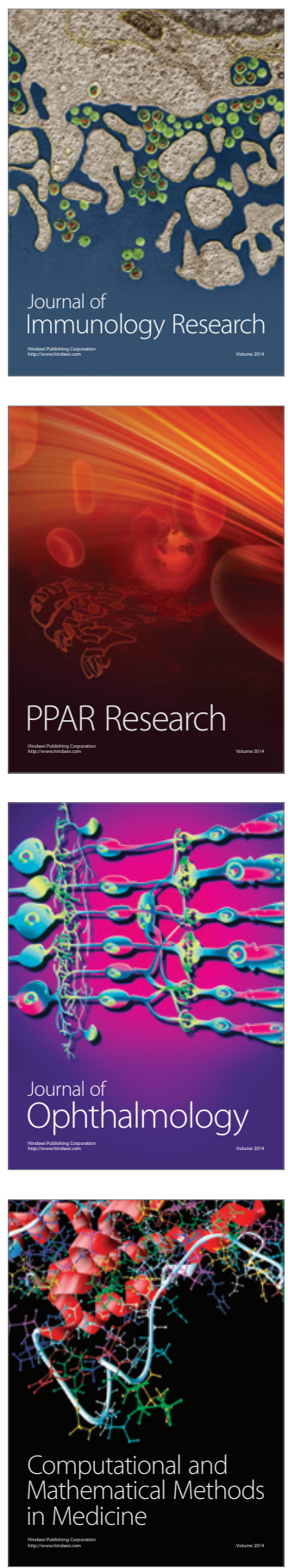

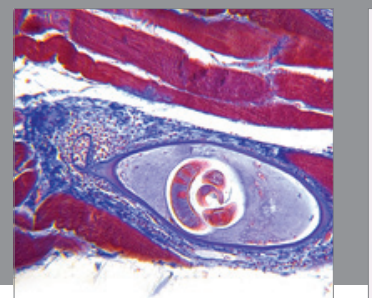

Gastroenterology

Research and Practice
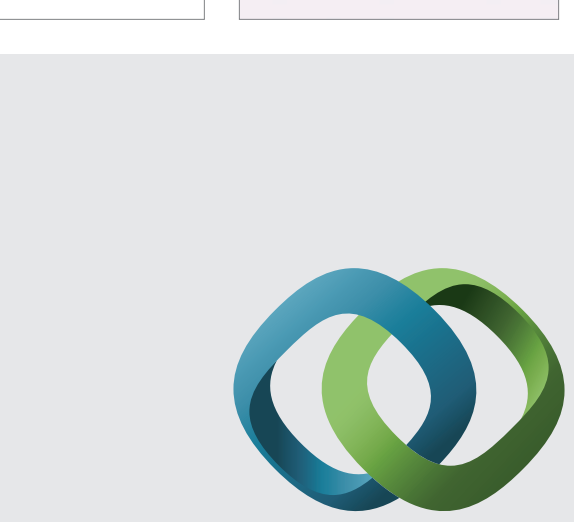

\section{Hindawi}

Submit your manuscripts at

http://www.hindawi.com
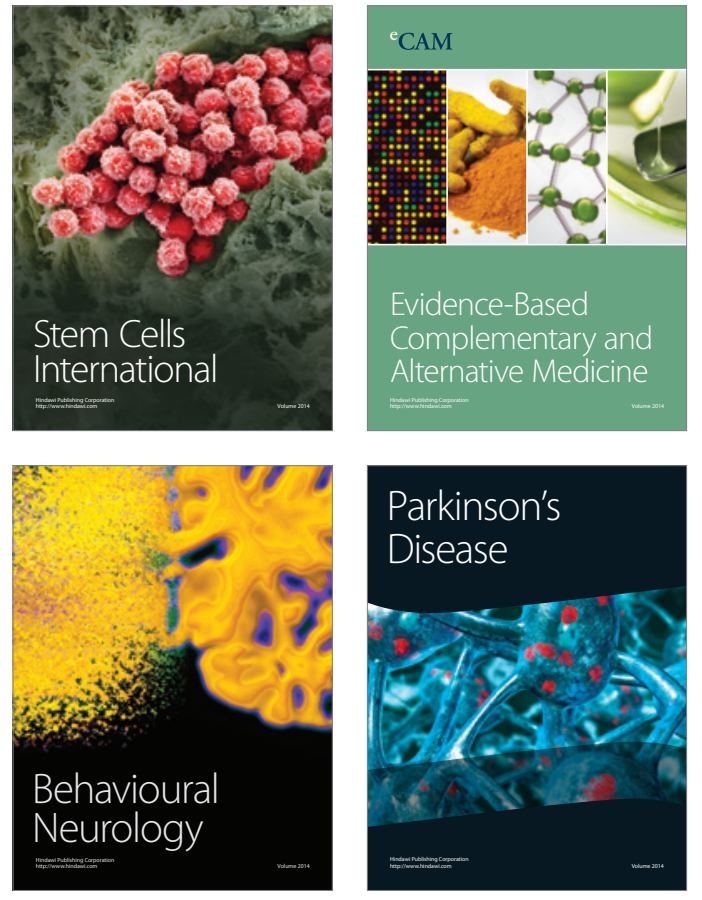
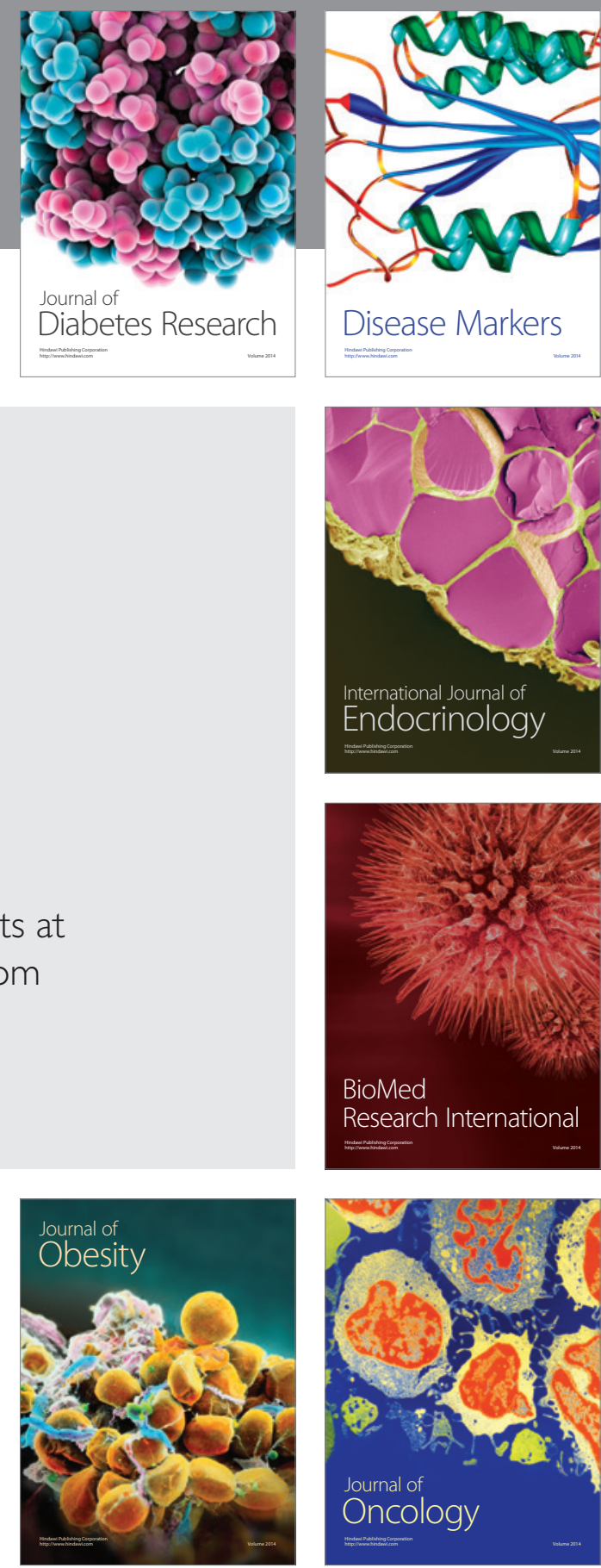

Disease Markers
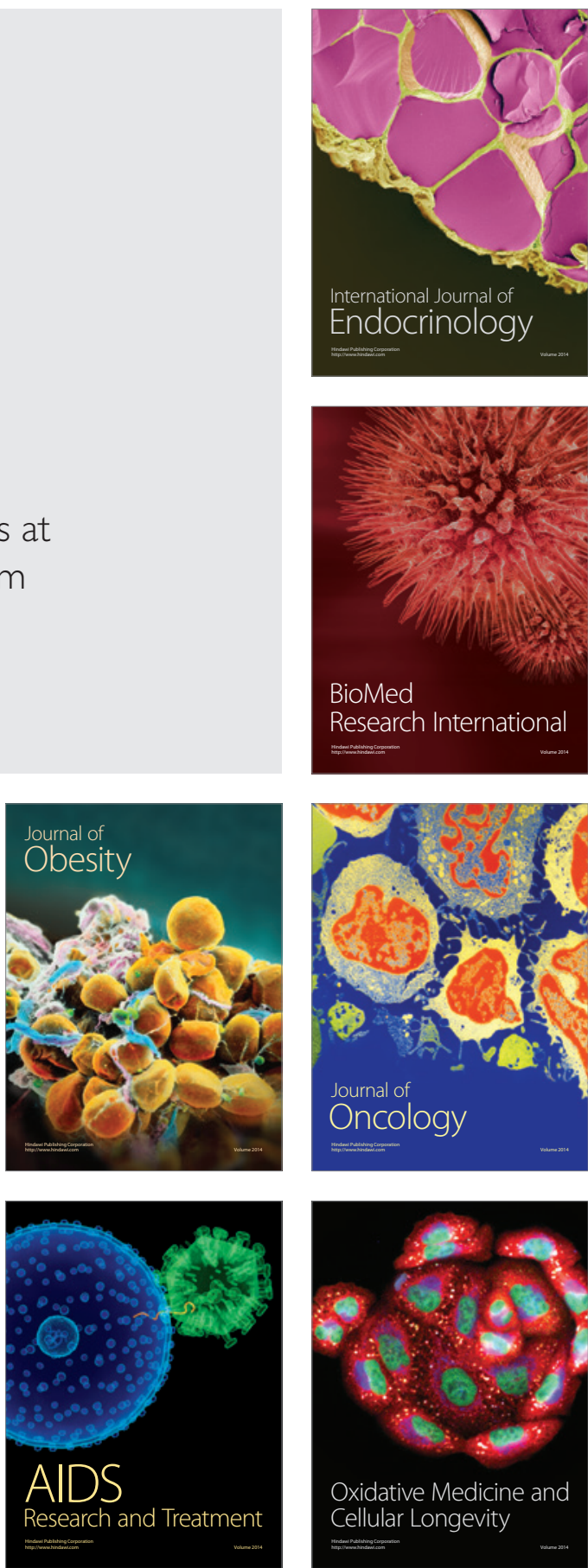